\title{
Répartition de la biomasse entre organes végétatifs et reproducteurs chez le hêtre européen (Fagus sylvatica L), selon le secteur de la couronne et l'âge des arbres
}

\author{
B Comps 1, B Thiébaut 2, G Barrière 1, J Letouzey 1
}

1 Université de Bordeaux l, département de biologie des végétaux ligneux, laboratoire d'écologie génétique, avenue des Facultés, 33405 Talence cedex;

2 Université des sciences et techniques du Languedoc, Institut de botanique, 163, rue A Broussonnet, 34000 Montpellier;

CNRS, centre d'écologie fonctionnelle et évolutive, BP 5051, 34033 Montpellier cedex, France

(Reçu le 28 décembre 1992; accepté le 17 septembre 1993)

\begin{abstract}
Résumé - Les allocations de ressources chez les végétaux peuvent être estimées pour étudier la répartition de la biomasse entre différents organes lors d'un cycle de végétation annuel. Quatorze hêtres (Fagus sylvatica $L$ ) ont été étudiés : 8 arbres très âgés (150 ans environ) et 6 arbres adultes de 100 ans environ. La couronne de chaque arbre a été divisée en 2 secteurs, supérieur et inférieur. Le hêtre produit 2 types de pousses annuelles, longues et courtes. Elles peuvent être végétatives ou florifères. Des pousses de chaque type situées sur des axes de premier ordre dans la ramification ont été analysées pour décrire la répartition de la matière sèche entre différentes fonctions végétatives et reproductrices. Les stratégies de développement changent selon l'âge des arbres, le secteur et les types de pousses. Les fonctions végétatives sont favorisées dans les pousses longues des arbres jeunes, surtout dans le secteur supérieur de la couronne. Les fonctions reproductrices mobilisent une biomasse plus importante chez les arbres âgés que chez les arbres plus jeunes. Chez tous les arbres, l'allocation de matière reste élevée pour assurer la maintenance dans les pousses végétatives courtes, ce qui confirme leur rôle essentiel d'exploitation du milieu.
\end{abstract}

allocation de ressources / pousse annuelle / secteur du houppier / âge / Fagus sylvatica

Summary - Biomass distribution in vegetative and reproductive organs of the European beech (Fagus sylvatica L), according to crown sector and tree age. Resource allocation in plants can be estimated to study biomass distribution in the various organs during an annual vegetative cycle. Fourteen beech trees were chosen on the Aigoual mountain (Cévennes, France, $44^{\circ}$ $20 ' \mathrm{~N}, 3^{\circ} 60^{\prime} \mathrm{E}$, alt $1400 \mathrm{~m}$ ): 8 were very old trees (150 yr), and 6 were vigorous adult trees (100 yr old). The tree crown was divided into upper and lower sectors. Beech produces long (L) and short (C) annual shoots, which are vegetative (V) or both vegetative and floriferous. Shoots of each type located on first-order axes in the ramification were analysed to describe the distribution of dry matter among various vegetative and reproductive functions. In each tree, 40 terminal buds were chosen at random: 20 on the upper part of the crown and 20 on the lower sector; approximately half seemed 
floriferous. During April these buds were enclosed in situ in gauze bags permeable to pollen and light. The 640 shoots produced from these buds were collected during the following November; of these only 524 were in good condition and were analysed. After desiccation, shoot length (V2L) was measured and shoot organs were weighed: leaves and stipules (V1), axes (V2P), buds (V3), female production (R1) and male inflorescences (R2). Development strategies vary according to tree age, tree sector and shoot type. Vegetative functions are favoured in long shoots of the youngest trees particularly in the upper sector (except V1). In short shoots, biomass is also more important in the upper crown sector. There was no biomass difference in hermaphrodite shoots according to the sector. But the age effect is considerable: female acquisitions are much greater in old trees $(p<0.001)$, whereas male acquisitions tend to be a little lower $(\mathrm{p}<0.05)$. There are great differences in vegetative biomass according to shoot type, but, in hermaphrodite shoots there are few differences in reproductive functions according to age and sector. Biomass allocation (\% of the shoot biomass) is often different according to tree age: (i) female allocation in hermaphrodite shoots is much greater in the oldest trees, whereas it is the opposite for vegetative functions; and (ii) vegetative long-shoot allocation is greatest for the $V 1$ function in old trees, whereas vegetative short shoot allocation is very high for the $\checkmark 1$ function whatever the age (this confirms their essential role in exploiting the environment). There are significant positive biomass correlations in vegetative functions, above all in long shoots. Concerning biomass allocations, correlations among vegetative functions vary according to age and shoot type. Generally, there is a negative correlation between female allocation and the various vegetative allocations. All these results show: (i) a partial development of each shoot type; (ii) a synergy between vegetative functions in hermaphrodite shoots (biomass and allocations), whereas a strong competition appears for allocations principally between female and vegetative functions; and (iii) a tree age effect: in $100 \mathrm{yr}$-old trees, vegetative development is still very important, principally at the upper part of the crown. In very old trees, vegetative development tends to become stable and female function is preponderant.

resource allocation / annual shoot / crown sector / tree age / Fagus sylvatica

\section{INTRODUCTION}

Les allocations de ressources chez les végétaux peuvent être étudiées en examinant la répartition de la biomasse et/ou celle des éléments nutritifs entre différents organes lors d'un cycle de végétation. Les ressources analysées changent selon les objectifs des études qui peuvent être d'ordre morphologique et architectural (Benner et Bazzaz, 1988 ; Wall et Morrison, 1990) ou physiologique (Williams et Bell, 1981; Abrahamson et Mc Crea, 1985 ; Staaf et Jernquist, 1986). Les auteurs comparent parfois la répartition des allocations entre organes aériens et souterrains du végétal (Munson et Timmer, 1990) ou bien, dans les organes aériens, entre fonctions végétatives et reproductrices (Tuomi et al, 1982; Caeser et Mac Donald, 1983 ; Rameau, 1986 ; Gart- ner, 1988; Escarré et Houssard, 1989 ; Thiébaut et Comps, 1991). Un autre objectif est celui de comprendre l'influence de facteurs du milieu sur la biomasse et l'allocation de cette biomasse et des nutriments aux différents organes du végétal (Menges, 1990 ; Munson et Timmer, 1990 ; Van Baalen et al, 1990; Wall et Morrison, 1990; Wankhar et Tripathi, 1990).

Le hêtre européen produit des pousses annuelles, longues ( $L$ ) ou courtes (C), végétatives $(\mathrm{V})$ ou à la fois végétatives et florifères. Ces dernières peuvent être hermaphrodites ou unisexuées, mâles ou femelles, et elles seront ainsi distinguées dans la suite de l'exposé. Les pousses Iongues ont un rôle essentiellement charpentier et explorent le milieu alors que les pousses courtes contribuent à augmenter la surface chlorophylienne et exploitent le 
milieu (Thiébaut et al, 1981; Thiébaut, 1982 ; Thiébaut et Puech, 1983, 1984). Thiébaut et Comps (1991) ont montré que les pousses florifères mobilisent des ressources plus importantes que les pousses végétatives. De plus, une partie importante de leurs ressources est employée à la reproduction mais leur stratégie diffère selon leur type: dans les pousses courtes, un apport plus important de ressources bénéficie à la fois aux fonctions végétatives et reproductrices alors que dans les pousses longues une compétition se dessine entre ces 2 fonctions. Ces résultats reposent seulement sur l'analyse de 6 individus adultes isolés, tous du même âge et sans distinction de secteurs dans le houppier. Or Thiébaut et al (1981) décrivent plusieurs zones dans la couronne d'un hêtre adulte, avec des modalités de croissance différentes : zone en exploration à croissance végétative importante vers le haut de la couronne et zone en exploitation à croissance réduite vers le bas. Et chez un arbre âgé, la zone en exploration se réduit puis disparait, cédant la place à une zone en exploitation vers le haut et une zone d'élagage vers le bas.

L'objectif de ce travail est double : (i) contrôler les résultats précédents par l'étude d'un plus grand nombre d'individus, et (ii) mieux comprendre la stratégie de développement, par l'introduction de 2 facteurs de variation possible pour les allocations de biomasse dans les pousses annuelles: le secteur et l'âge de l'arbre.

\section{MÉTHODES}

\section{Dispositif expérimental et mesures}

En 1988, 14 arbres ont été pris au hasard dans le massif de l'Aigoual (Cévennes, France, $44^{\circ} 20 \mathrm{~N}, 3^{\circ} 60 \mathrm{E}$ ), 8 dans une station (La Dauphine), 6 dans une autre (Plo du Four), très proches l'une de l'autre, toutes 2 localisées sur le même substrat, à des altitudes voisines (1 $400 \mathrm{~m}$ environ) et présentant donc des conditions écologiques tout à fait similaires. L'effet station peut donc être considéré comme négligeable et les écarts observés entre les deux groupes d'individus sont alors dus essentiellement aux différences d'âge des arbres. Le peuplement est de densité moyenne en sorte que la partie supérieure des houppiers est soumise à un éclairement plus important que leur base. Dans la première station, les arbres sont âgés de 100 ans environ et encore en pleine croissance, dans la seconde ils sont plus vieux, de l'ordre de 150 ans. Sur chaque arbre, 40 bourgeons terminaux ont été retenus au hasard sur tout le pourtour de la couronne : 20 au sommet (secteur haut) et 20 dans les zones inférieure et médiane (secteur bas), en se basant sur des critères de développement des axes et de grosseur des bourgeons (Brett, 1963; ColomboMariani, 1971) permettant d'optimiser la probabilité d'obtenir un nombre équivalent des 4 types de pousses possibles : longues végétatives (LV), longues florifères courtes végétatives (CV) et courtes florifères. L'étude des bourgeons du secteur haut a nécessité l'utilisation d'une nacelle hydraulique. Les bourgeons étaient tous situés à la périphérie des arbres, à l'extrémité de branches maîtresses de rang 2 dans la ramification. Au printemps, avant la feuillaison, chaque bourgeon a été enfermé dans un sac de gaze perméable au pollen, suffisamment grand pour permettre le développement normal de la pousse. Au mois de novembre suivant, les sacs ont été prélevés avec leur contenu, correspondant à tous les organes produits au cours d'un cycle annuel de végétation.

Sur l'ensemble des 640 pousses du dispositif, 524 seulement étaient en bon état et ont pu être utilisées. Leurs effectifs selon le type, la station et le secteur apparaissent dans les tableaux I et III. Après dessiccation pendant 5 jours à $80^{\circ}$, le poids étant alors stabilisé, la longueur des axes a été mesurée et les organes de chaque pousse ont été pesés, en séparant : les feuilles et les stipules $(V 1$, fonction de maintenance), les axes (V2, croissance actuelle : $V 2 P$, biomasse, $\mathrm{V} 2 \mathrm{~L}$, longueur), les bourgeons ( $\mathrm{V} 3$, croissance future), les infrutescences (R1, reproduction femelle) et les inflorescences mâles ( $R 2$, reproduction mâle).

La répartition progressive de la biomasse au cours de l'année n'a pas été analysée à cause 
de la hauteur des arbres, comme ont pu le faire d'autres auteurs chez des espèces de petite taille (Harper et Ogden, 1970; Dina et Klikoff, 1974 ; Pitelka, 1977 ; Bell et al, 1979). Les allocations à la floraison femelle n'ont pas été mesurées avant la fructification comme le préconise Agren (1988). Pour la reproduction mâle, la masse du pollen n'a pas été comptabilisée bien qu'elle représente jusqu'à $15 \%$ du poids sec des inflorescences du hêtre (Nielsen, 1977; Oswald, 1981). On peut considérer que la production de faînes était bonne au cours de l'année d'observation, ce qui autorise la comparaison entre allocations aux fonctions reproductrices et végétatives.

\section{Traitement des données}

Le traitement des données a été réalisé en tenant compte des valeurs absolues de biomasse ou de longueur des axes (acquisitions en mg de matière sèche ou en $\mathrm{mm}$ ) et du poids relatif pour chaque fonction par rapport au poids total de la pousse (allocations en \%) (Hickman et Pitelka, 1975 ; Jacquard et al, 1985).

Des analyses de variance à 2 facteurs croisés (Baradat, 1989, résultats non reportés) n'ont mis en évidence que de rares interactions secteur-arbres et âge-secteur, ce qui autorise toutes les comparaisons effectuées sur les moyennes. La distribution des variables étudiées n'étant pas toujours normale, les comparaisons de moyennes ont été effectuées à l'aide du test non paramétrique de Mann-Whitney.

\section{RÉSULTATS}

\section{Acquisitions}

\section{Influence du secteur et de l'âge des arbres}

L'effet secteur est significatif surtout dans les pousses longues pour les différentes fonctions végétatives sauf $V_{1}$ dans les pousses florifères et uniquement à $\mathrm{La}$ Dauphine (arbres de 100 ans) où le secteur haut présente une production plus élevée (tableau I). C'est pour les pousses végétatives longues que l'écart de production végétative entre les arbres des 2 stations est le plus élevé. Dans les pousses végétatives courtes l'effet secteur n'est significatif qu'au Plo du Four (arbres de 150 ans, tableau I) avec une production plus élevée dans le secteur haut.

Les pousses hermaphrodites investissent à peu près de la même manière dans les 2 secteurs pour les fonctions reproductrices dans chaque groupe d'arbres (tableau I). Mais l'effet «âge» est considérable : les acquisitions à la fonction femelle sont supérieures au Plo du Four $(P<$ 0.001 ) et celles à la fonction mâle ont tendance à être un peu plus fortes à La Dauphine $(P<0,05)$.

Dans les pousses unisexuées, il n'y a pas d'écart important inter-secteur ou interâge pour la fonction mâle (tableau I). Pour les pousses femelles, une différence apparaît entre groupes d'arbres: à La Dauphine, les pousses longues ne sont présentes que dans le secteur haut, avec des acquisitions à la reproduction très faibles et une biomasse végétative élevée; au Plo du Four les 2 types de pousses femelles sont présents dans les 2 secteurs, avec des acquisitions à la reproduction plus élevées et une production végétative plus faible.

\section{Comparaison entre les différents types de pousses}

La quantité de matière mise en œuvre dans les fonctions végétatives est significativement plus élevée dans les pousses tongues que dans les pousses courtes, Iorsqu'on compare séparément les pousses végétatives et les pousses hermaphrodites, quels que soient l'âge et le secteur (tableau 11). En revanche, les écarts ne sont pas significatifs pour la matière employée dans les fonctions repro- 
Tableau I. Acquisitions selon le type de pousses et comparaison des moyennes entre secteurs selon l'âge des arbres (test de Mann-Whitney).

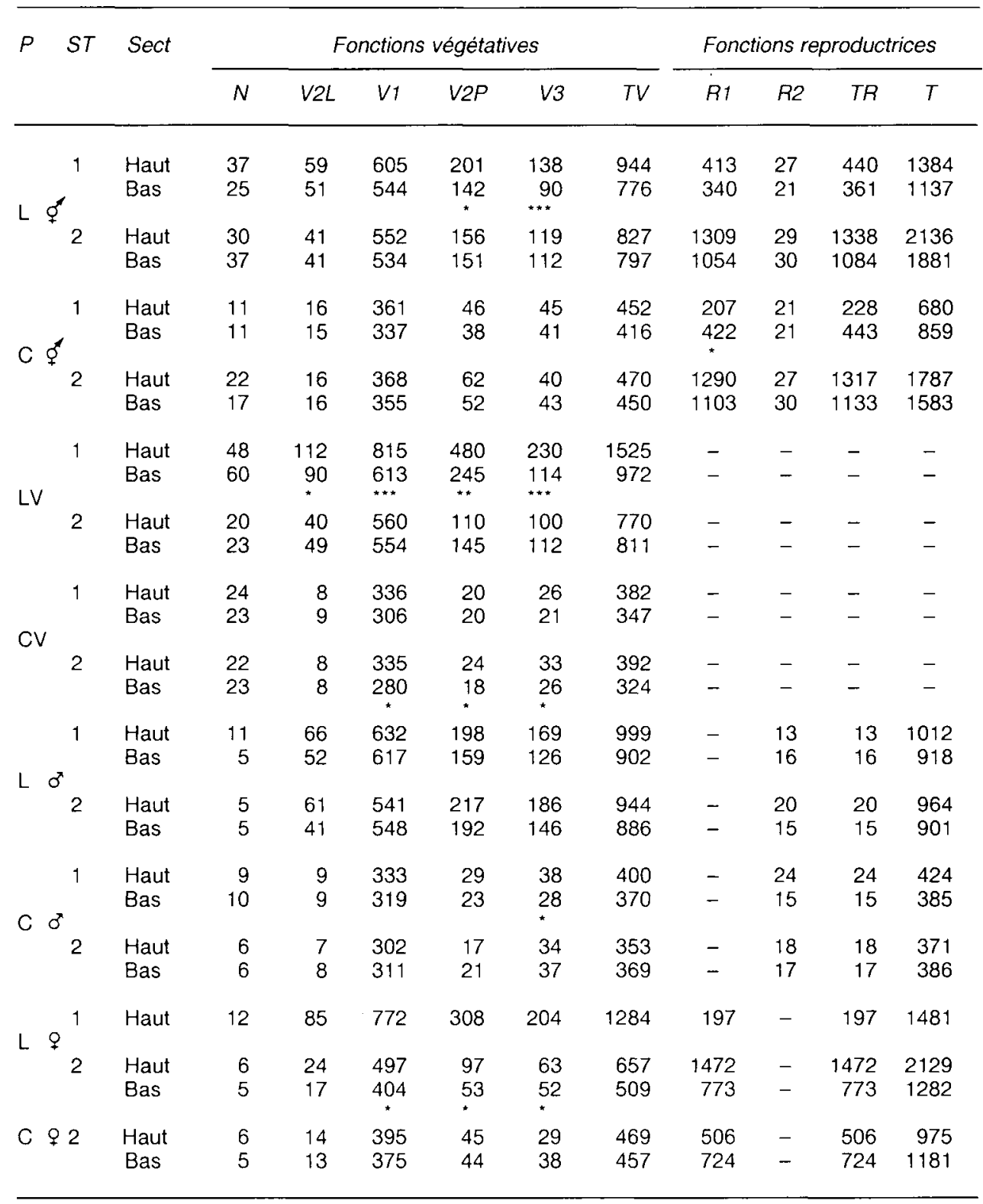

ST1 : arbres de la Dauphine, ST2 : vieux arbres du Plo du Four. Pousses (P) : V, végétatives ; L, longues ; C, courtes. Acquisitions ( $\mathrm{mg}$ et $\mathrm{mm}$ ) : V1, feuilles ; V2P et $\mathrm{V} 2 \mathrm{~L}$, masse et longueur des axes ; $\mathrm{V} 3$, bourgeons ; R1, production femelle; R2, production mále ; TV, total de la biomasse végétative ; TR, total de la biomasse reproductrice ; $\mathrm{T}$, total de la biomasse. Seuls ont été notés les écarts significatifs : ${ }^{\star \star *}, P<0.001 ;{ }^{* *}, 0.001<P<0.01 ;{ }^{*}, 0.01<P$ $<0.05$. 
Tableau II. Comparaison des acquisitions entre différents types de pousses, pour chaque fonction, selon l'âge et les secteurs (test de Mann-Whitney).

\begin{tabular}{|c|c|c|c|c|c|c|c|c|}
\hline \multirow[t]{2}{*}{ Pousses } & \multirow[t]{2}{*}{$\hat{A} g e$} & \multirow[t]{2}{*}{ Secteur } & \multicolumn{6}{|c|}{ Fonctions } \\
\hline & & & $V_{1}$ & $V 2 P$ & V2L & $V 3$ & $R 1$ & $R 2$ \\
\hline & 1 & Haut & ** & ** & 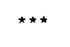 & * & - & - \\
\hline LV & & Bas & ns & $*$ & $* * *$ & $\mathrm{~ns}$ & - & - \\
\hline \multirow[t]{2}{*}{$L \phi^{x}$} & 2 & Haut & ns & + & $\mathrm{ns}$ & $\mathrm{ns}$ & - & - \\
\hline & & Bas & ns & ns & ns & ns & - & - \\
\hline \multirow[b]{2}{*}{$c q^{\sigma}$} & 1 & Haut & ns & $* * *$ & $\star \star *$ & $* *$ & - & - \\
\hline & & Bas & ns & $\star \star \star *$ & $*$ & * & - & - \\
\hline \multirow{3}{*}{$\mathrm{CV}^{\wedge}$} & & & & & & & & \\
\hline & 2 & Haut & ns & *** & $\star \star \star \star$ & ns & - & - \\
\hline & & Bas & $*$ & $* *$ & 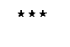 & $* * *$ & - & - \\
\hline \multirow[b]{2}{*}{ LV } & 1 & Haut & $* \star \star$ & $* * *$ & $\star \star \star *$ & $* * *$ & - & - \\
\hline & & Bas & $\star \star \star \star$ & $* * *$ & $\star \star *$ & 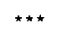 & - & - \\
\hline \multirow{3}{*}{$c v^{x}$} & & & & & & & & \\
\hline & 2 & Haut & $\star \star \star *$ & $* * *$ & $\star * *$ & $* * *$ & - & - \\
\hline & & Bas & 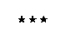 & 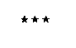 & $\star \star \star *$ & $* * *$ & - & - \\
\hline \multirow{2}{*}{ L ${ }_{f}$} & 1 & Haut & $\star \star \star *$ & $* * *$ & ${ }_{\star \star \star}$ & $\star \star \star *$ & * & - \\
\hline & & Bas & $\star \star \star \star ⿱ 乛 ⿻ 上 丨$ & $\star \star \star$ & $* * \star$ & 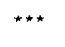 & ns & ns \\
\hline \multirow[t]{2}{*}{$c q^{x}$} & 2 & Haut & $* * *$ & $\ldots$ & $* * *$ & $* * *$ & ns & ns \\
\hline & & Bas & $\star \star \star *$ & $\star * *$ & $\star * \star *$ & $* * *$ & ns & ns \\
\hline
\end{tabular}

Pousses : $V$, végétatives ; $L$, longues ; $C$, courtes. Acquisitions (mg et $\mathrm{mm}): V 1$, feuilles ; $V 2 P$ et $V 2 L$, masse et longueur des axes ; V3, bourgeons ; R1, production femelle; R2, production mâle. Âge : 1, arbres de La Dauphine ; 2 , vieux arbres du Plo du Four. ${ }^{* *} P<0,001 ;{ }^{* *} 0,001<P<0,01 ;+$ ou ${ }^{*} 0,01<P<0,05$; ns : non significatif. Le symbole * est utilisé lorsque, pour une comparaison donnée, les acquisitions dans le premier type de pousse mentionné sont supérieures au second ; le symbole + est utilisé dans les cas contraires.

ductrices entre les pousses longues et courtes hermaphrodites.

Dans les pousses longues la quantité totale de matière mobilisée ne diffère pas de manière significative entre les pousses hermaphrodites et végétatives à La Dauphine alors qu'elle est beaucoup plus élevée chez les hermaphrodites au Plo du Four (tableau I, résultats du test non mentionnés sur le tableau). Autrement dit, chez les arbres en pleine vitalité, les res- sources employées pour les fonctions végétatives sont plus faibles dans les pousses hermaphrodites que dans les végétatives, les premières utilisant une partie de leurs ressources pour les fonctions reproductrices. Chez les vieux arbres, la quantité de matière employée aux fonctions végétatives est comparable dans les 2 types de pousses.

Dans les pousses courtes, la biomasse mise en œuvre est plus élevée dans les 
hermaphrodites que dans les végétatives pour toutes les fonctions végétatives sauf pour la fonction V1 (tableau I).

Les pousses longues, mâles et hermaphrodites, ne présentent pas de différence nette (tableau I). Les pousses courtes mâles mobilisent moins de matière végétative que les hermaphrodites et, de ce fait, se comportent sensiblement comme des pousses végétatives courtes. Les pousses longues femelles se comportent différemment selon l'âge des arbres: chez les arbres en pleine croissance, elles sont comparables aux longues végétatives; chez les vieux arbres, la fonction femelle n'est pas différente de celles des longues hermaphrodites, et leur biomasse aux différentes fonctions végétatives est intermédiaire entre celle des pousses hermaphrodites longues et courtes. Les pousses courtes femelles ne sont présentes que sur les vieux arbres et ne diffèrent des pousses courtes que par une production femelle un peu inférieure. Toutes ces comparaisons prenant en compte les pousses unisexuées ont été testées à l'aide du test de Mann-Whitney mais n'ont pas été reportées sur le tableau II.

\section{Allocations}

\section{Influence du secteur et de l'âge des arbres}

Quelques écarts apparaissent entre secteurs, en particulier dans les pousses Iongues des arbres en pleine croissance où l'allocation à la maintenance (V1) est la plus élevée dans les secteurs bas, alors qu'elle est la plus faible pour la croissance future (V3) (tableau III). La plupart des différences entre les 2 groupes d'arbres sont significatives (résultats des tests non reportés sur le tableau) et il faut distinguer (tableau III) :
- les pousses hermaphrodites qui allouent beaucoup plus à la fonction femelle chez les vieux arbres que chez les arbres en pleine croissance, alors qu'on observe l'inverse pour les fonctions végétatives. II en résulte un renversement complet des tendances selon l'âge des arbres;

- les pousses végétatives longues qui allouent davantage à la maintenance et moins à la croissance chez les vieux arbres et les pousses végétatives courtes pour lesquelles les différences sont moins marquées et qui allouent massivement à la maintenance quel que soit l'âge.

Les allocations dans les fleurs mâles varient peu d'un secteur à l'autre et entre les 2 groupes d'arbres. Les pousses femelles longues investissent fortement dans la fonction R1 chez les vieux arbres et surtout dans le secteur haut. Les pousses courtes allouent autant dans les fonctions végétatives (surtout la maintenance) que dans la reproduction femelle.

\section{Comparaison entre les différents types de pousses}

Dans les pousses végétatives, l'allocation à la maintenance $(\mathrm{V} 1 / \mathrm{T})$ est beaucoup plus élevée dans les pousses courtes alors que l'allocation à la croissance (V2P/T et V $3 / T$ ) est plus grande dans les pousses longues (tableau IV).

Dans les pousses hermaphrodites, l'effort est prépondérant pour la croissance dans les pousses longues et pour les fonctions reproductrices dans les pousses courtes. L'allocation à la maintenance est équivalente dans ces 2 types de pousses.

Les pousses florifères unisexuées présentent des comportements différents d'un type à l'autre (tableau III). L'allocation à la reproduction est faible dans les pousses longues mâles, en sorte que la distribution de leur biomasse se rapproche le plus de celle des pousses longues végétatives. 
Tableau III. Allocations selon le type de pousses et comparaison des moyennes entre secteurs pour les 2 groupes d'arbres (test de Mann-Whitney).

\begin{tabular}{|c|c|c|c|c|c|c|c|c|c|c|}
\hline \multirow[t]{2}{*}{$P$} & \multirow[t]{2}{*}{$S T$} & \multirow[t]{2}{*}{ Sect } & \multicolumn{5}{|c|}{ Fonctions végétatives } & \multicolumn{3}{|c|}{ Fonctions reproductrices } \\
\hline & & & $N$ & $V 1 / T$ & $V 2 P / T$ & $V 3 / T$ & $T V / T$ & $R 1 / T$ & $R 2 / T$ & $T R / T$ \\
\hline \multirow{4}{*}{ L $\Phi$} & 1 & Haut & 37 & 48,0 & 13,9 & 10,6 & 72,5 & 25,1 & 2,4 & 27,5 \\
\hline & & Bas & 25 & 52,6 & 12,1 & 8,1 & 72,8 & 25,3 & 1,9 & 27,2 \\
\hline & 2 & Haut & 30 & 30,3 & 7,6 & 6,3 & 44,2 & 54,1 & 1,7 & 55,8 \\
\hline & & Bas & 37 & 33,3 & 7,9 & 6,6 & 47,8 & 50,4 & 1,8 & 52,2 \\
\hline \multirow{4}{*}{ C $\$$} & 1 & Haut & 11 & 57,3 & 6,8 & 7,1 & 71,2 & 25,6 & 3,2 & 28,8 \\
\hline & & Bas & 11 & 44,4 & 4,6 & 4,6 & 53,6 & 43,6 & 2,8 & 46,4 \\
\hline & 2 & Haut & 22 & 30,3 & 4,2 & 3,4 & 37,9 & 60,0 & 2,1 & 62,1 \\
\hline & & Bas & 17 & 27,7 & 3,7 & 3,4 & 34,8 & 63,1 & 2,1 & 65,2 \\
\hline \multirow{4}{*}{ LV } & 1 & Haut & 48 & 60,9 & 25,0 & 14,1 & - & - & - & - \\
\hline & & Bas & 60 & 66,9 & 22,2 & 10,9 & - & - & - & - \\
\hline & 2 & Haut & 20 & 75,0 & 12.9 & 12.1 & _- & _- & _- & - \\
\hline & & Bas & 23 & 72,7 & 14,5 & 12,8 & - & - & - & - \\
\hline \multirow{4}{*}{ CV } & 1 & Haut & 24 & 87,9 & 5,2 & 6,9 & - & - & - & - \\
\hline & & Bas & 23 & 88,1 & 5,7 & 6,2 & - & - & - & - \\
\hline & 2 & Haut & 22 & 85,0 & 6,0 & 9,0 & - & - & - & - \\
\hline & & Bas & 23 & 86,4 & 5,5 & 8,1 & - & - & - & - \\
\hline \multirow{4}{*}{ L $\quad 0^{x}$} & 1 & Haut & 11 & 62,2 & 18,5 & 14,9 & 98,6 & - & 1,4 & 1,4 \\
\hline & & Bas & 5 & 68,8 & 16,0 & 13,1 & 97,9 & - & 2,1 & 2,1 \\
\hline & 2 & Haut & 5 & 59,0 & 19,9 & 19,3 & 98,2 & - & 1,8 & 1,8 \\
\hline & & Bas & 5 & 67,9 & 14,9 & 15,9 & 98,7 & - & 1,3 & 1,3 \\
\hline \multirow{4}{*}{ C $0^{\pi}$} & 1 & Haut & 9 & 79,2 & 6,6 & 7,6 & 93,4 & - & 6,6 & 6,6 \\
\hline & & Bas & 10 & 82,0 & 5,8 & 8,0 & 95,8 & - & 4,2 & 4,2 \\
\hline & 2 & Haut & 6 & 802 & 45 & 98 & 94.5 & _- & 5.5 & 55 \\
\hline & & Bas & 6 & 79,4 & 5,3 & 10,3 & 95,0 & - & 5,0 & 5,0 \\
\hline \multirow{3}{*}{$L q$} & 1 & Haut & 12 & 54,5 & 19,1 & 13,3 & 88,9 & 11,1 & - & 11,1 \\
\hline & 2 & Haut & 6 & 23,7 & 4,4 & 3,1 & 31,2 & 68,8 & - & 68,8 \\
\hline & & Bas & 5 & 36,4 & 5,7 & 6,2 & 48,3 & 51,7 & - & 51,7 \\
\hline \multirow[t]{2}{*}{ C 9} & 2 & Haut & 6 & 47,2 & 5,4 & 3,5 & 56,1 & 43,9 & - & 43,9 \\
\hline & & Bas & 5 & 37,3 & 4,5 & 4,5 & 46,3 & 53,7 & - & 53,7 \\
\hline
\end{tabular}

ST1 : arbres de la Dauphine ; ST2 : vieux arbres du Plo du Four. Pousses $(P): V$, végétatives ; $L$, longues ; C, courtes. Allocations \% : V1/T; feuilles ; V2P/T, masse des axes ; $V 3 / T$, bourgeons ; R1/T production femelle ; R2/T, production mâle ; TV/T, allocation végétative totale; TR/T, allocation reproductrice totale. Seuls ont été notés les écarts significatifs: ${ }^{* *} P<0,001 ;{ }^{* *} 0,001<P<0,01 ;{ }^{*} 0,01<P<0,05$. 
Tableau IV. Comparaison des allocations entre différents types de pousses, pour chaque fonction, selon l'âge des arbres et les secteurs (test de Mann-Whitney).

\begin{tabular}{|c|c|c|c|c|c|c|c|c|}
\hline \multirow[t]{2}{*}{ Pousses } & \multirow[t]{2}{*}{$S T$} & \multirow[t]{2}{*}{ Secteur } & \multicolumn{6}{|c|}{ Fonctions } \\
\hline & & & $V 1 / T$ & $V 2 P / T$ & - & $V 3 / T$ & $R 1 / T$ & $R 2 / T$ \\
\hline & 1 & Haut & + & $\cdots$ & - & * & ns & + \\
\hline L 9 & & Bas & $\mathrm{ns}$ & $\cdots+$ & - & $*$ & + & ns \\
\hline \multirow{3}{*}{$\begin{array}{l}x \\
\text { C } q\end{array}$} & & & & & & & & \\
\hline & 2 & Haut & ns & $\ldots * *$ & - & $\star \star \star \star$ & + & + \\
\hline & & Bas & ns & $\cdots *$ & - & $* \star \star$ & ++ & ns \\
\hline \multirow{5}{*}{$\begin{array}{l}\text { LV } \\
x \\
C V\end{array}$} & 1 & Haut & +++ & $\ldots$ & - & $* * *$ & - & - \\
\hline & & Bas & +++ & $* * *$ & - & 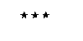 & - & - \\
\hline & & & & & & & & \\
\hline & 2 & Haut & $+t+$ & $\ldots * *$ & - & 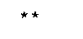 & - & - \\
\hline & & Bas & $t+t$ & $\cdots$ & - & $* \star *$ & - & - \\
\hline
\end{tabular}

Pousses : $V$, végétatives ; $L$, longues ; $C$, courtes. Allocations $\%: V 1 / T$, feuilles ; $V 2 P / T$ masse des axes ; $V 3 / T$; bourgeons ; R1/T, production femelle ; R2/T, production mâle. Åge : 1 , arbres de La Dauphine ; 2 , vieux arbres du Plo du Four. +++ ou ${ }^{* *} P<0,001 ;++$ ou ${ }^{*} 0,001<P<0,01 ;+$ ou $0,01<P<0,05 ;$ ns : non significatif. Le symbole est utilisé lorsque, pour une comparaison donnée, les allocations dans le premier type de pousse mentionné sont supérieures au second ; le symbole + est utilisé dans les cas contraires.

Les pousses courtes mâles, malgré un effort relativement important à la reproduction, accordent la priorité à la maintenance, comme des pousses végétatives courtes. Les allocations dans les pousses longues femelles se distribuent à peu près comme dans les longues hermaphrodites, malgré quelques écarts significatifs: en particulier, l'investissement dans la reproduction femelle est faible chez les arbres en pleine croissance et fort chez les vieux arbres, surtout dans le secteur haut. Les pousses courtes femelles des vieux arbres ressemblent le plus aux courtes hermaphrodites mais leur investissement est plus fort dans la maintenance $(\mathrm{V} 1 / \mathrm{T})$ et, au contraire, plus faible dans la reproduction femelle $(R 1 / T)$. Toutes ces comparaisons ont été testées à l'aide du test du MannWhitney, non reportées sur le tableau III.

\section{Corrélations entre les fonctions}

Dans cette analyse n'ont été prises en compte que les pousses végétatives et hermaphrodites aux effectifs suffisants. Des corrélations significatives et positives apparaissent entre les acquisitions aux fonctions végétatives dans les pousses longues, et surtout entre les fonctions $V 1$ et V2 dans les pousses courtes (tableau V). D'autre part, un effet "âge» ou sectoriel partiel apparaît au niveau des fonctions reproductrices : corrélation positive entre R 1 et V2 uniquement chez les vieux arbres, entre $R 1$ et $R 2$ dans les pousses longues hermaphrodites des secteurs bas et dans les pousses courtes uniquement chez les vieux arbres.

Pour les allocations, les corrélations entre fonctions végétatives varient selon 
Tableau V. Acquisitions : matrice des corrélations de Spearman entre les fonctions prises 2 à 2 dans les pousses hermaphrodites et végétatives.

\begin{tabular}{|c|c|c|c|c|c|c|c|c|c|c|c|c|c|}
\hline \multirow[t]{2}{*}{ Fonctions } & \multirow[t]{2}{*}{ Åge } & \multirow[t]{2}{*}{ Secteurs } & \multicolumn{2}{|c|}{ Plongues } & \multicolumn{2}{|c|}{$P$ courtes } & \multirow[t]{2}{*}{ Fonctions } & \multirow[t]{2}{*}{ Åge } & \multirow[t]{2}{*}{ Secteurs } & \multicolumn{2}{|c|}{ Plongues } & \multicolumn{2}{|c|}{$P$ courtes } \\
\hline & & & 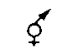 & v & $\Phi$ & V & & & & $\$$ & V & $\phi$ & V \\
\hline \multirow{5}{*}{$V_{1} \times V_{2} P$} & 1 & Haut & +++ & $++t$ & +++ & t+ & \multirow{5}{*}{$\mathrm{V} 2 \mathrm{~L} \times \mathrm{V} 3$} & 1 & Haut & +++ & +++ & ns & ns \\
\hline & & Bas & +++ & +++ & $\mathrm{ns}$ & + & & & Bas & + & +++ & + & ns \\
\hline & & & & & & & & & & & & & \\
\hline & 2 & Haut & +++ & ++ & +++ & +++ & & 2 & Haut & +++ & +++ & ns & ns \\
\hline & & Bas & +++ & +++ & ++ & +++ & & & Bas & +++ & +++ & ns & + \\
\hline \multirow{4}{*}{$V_{1} \times V_{2} L$} & 1 & Haut & +++ & +++ & + & +++ & \multirow{4}{*}{$\mathrm{V} 2 \mathrm{P} \times \mathrm{V} 2 \mathrm{~L}$} & 1 & Haut & +++ & +++ & ++ & +++ \\
\hline & & Bas & +++ & +++ & $\mathrm{ns}$ & + & & & Bas & ++ & +++ & ++ & +++ \\
\hline & 2 & Haut & ++ & ++ & +++ & + & & 2 & Haut & +++ & +++ & +++ & $+t+$ \\
\hline & & Bas & +++ & +++ & $\mathrm{ns}$ & ++ & & & Bas & +++ & +++ & ++ & +++ \\
\hline \multirow{5}{*}{$V 1 \times V 3$} & 1 & Haut & +++ & +++ & ns & ns & \multirow{5}{*}{$\mathrm{V} 2 \times \mathrm{R} 1$} & 1 & Haut & ns & & ns & \\
\hline & & Bas & + & +++ & ns & ns & & & Bas & ns & & ns & \\
\hline & & & & & & & & & & & & & \\
\hline & 2 & Haut & ++ & t+ & $\mathrm{ns}$ & ns & & 2 & Haut & ++ & & +++ & \\
\hline & & Bas & +++ & +++ & ++ & ++ & & & Bas & + & & ++ & \\
\hline \multirow{5}{*}{$V_{2} P \times V 3$} & 1 & Haut & +++ & +++ & $\mathrm{ns}$ & ns & \multirow{5}{*}{$\mathrm{R} 1 \times \mathrm{R} 2$} & 1 & Haut & ns & & ns & \\
\hline & & Bas & ++ & +++ & ns & ns & & & Bas & ++ & & ns & \\
\hline & & & & & & & & & & & & & \\
\hline & 2 & Haut & $++t$ & +++ & $\mathrm{ns}$ & ns & & 2 & Haut & ns & & ++ & \\
\hline & & Bas & +++ & +++ & ++ & ns & & & Bas & + & & +++ & \\
\hline
\end{tabular}

Pousses $(P): V$, végétatives. Fonctions : V1, feuilles; V2P et V2L, masse et longueur des axes; V3, bourgeons ; R1, production femelle ; R2, production mâle. Åge : 1, arbres de 100 ans de La Dauphine ; 2 , vieux arbres du Plo du Four. Toutes les corrélations significatives sont positives : $+++P<0,001 ;++0,001<P<0,01 ;+0,01<P<0,05$; ns : non significatif.

l'âge et le type de pousse (tableau VI). Ces fonctions sont souvent corrélées, positivement dans les pousses hermaphrodites et négativement (sauf $\mathrm{V} 2 \mathrm{P} / \mathrm{T} \times$ $V 3 / T$ ) dans les pousses végétatives. Dans les pousses courtes hermaphrodites les corrélations ne sont significatives que chez les vieux arbres. La fonction R1/T est corrélée négativement avec les 3 fonctions végétatives dans les pousses longues des 2 groupes d'arbres et dans les pousses courtes uniquement au Plo du Four. L'allocation mâle est corrélée positivement avec les 3 allocations végétatives dans le secteur haut des vieux arbres. Les allocations aux fonctions mâle et femelle sont corrélées négativement chez ces derniers. 
Tableau VI. Allocations : matrice des corrélations de Spearman entre les fonctions prises 2 à 2 dans les pousses hermaphrodites et végétatives.

\begin{tabular}{|c|c|c|c|c|c|c|c|c|c|c|c|c|c|}
\hline \multirow[t]{2}{*}{ Fonctions } & \multirow[t]{2}{*}{$\hat{A} g e$} & \multirow[t]{2}{*}{ Secteurs } & \multicolumn{2}{|c|}{ Plongues } & \multicolumn{2}{|c|}{$P$ courtes } & \multirow{2}{*}{ Fonctions } & \multirow[t]{2}{*}{$\hat{A} g e$} & \multirow[t]{2}{*}{ Secteurs } & \multicolumn{2}{|c|}{ Plongues } & \multicolumn{2}{|c|}{$P$ courtes } \\
\hline & & & $\phi$ & V & $\phi$ & $v$ & & & & $\phi$ & $v$ & $\Phi$ & $V$ \\
\hline \multirow{5}{*}{$\mathrm{V} 1 / \mathrm{T} \times \mathrm{V} 2 \mathrm{P} /$} & 1 & Haut & ns & $\star \star \star \star ~$ & ns & * & \multirow{5}{*}{$\mathrm{V} 2 \mathrm{P} / \mathrm{T} \times \mathrm{R} 1 /$} & 1 & Haut & 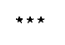 & & ns & \\
\hline & & Bas & + & $* * *$ & t+t & $\star \star *$ & & & Bas & 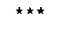 & & ns & \\
\hline & & & & & & & & & & & & & \\
\hline & \multirow[t]{2}{*}{2} & Haut & ++ & 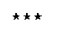 & +++ & * & & \multirow[t]{2}{*}{2} & Haut & $* * *$ & & $\star \star \star \star$ & \\
\hline & & Bas & +++ & $* \star *$ & +++ & $* *$ & & & Bas & $* \star *$ & & $* * *$ & \\
\hline \multirow{5}{*}{$V_{1} / T \times V_{3} / T$} & 1 & Haut & + & 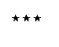 & ns & $\star \star \star ~$ & \multirow{5}{*}{$\mathrm{V} 3 / \mathrm{T} \times \mathrm{R} 1 / \mathrm{T}$} & 1 & Haut & 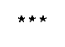 & & ns & \\
\hline & & Bas & ns & $* \star *$ & ns & $\star \star$ & & & Bas & ${ }^{\star \star}$ & & ns & \\
\hline & \multirow{3}{*}{2} & & & & & & & \multirow{3}{*}{2} & & & & & \\
\hline & & Haut & +++ & $* \star \star \star$ & t++ & $\star \star \star$ & & & Haut & $* * *$ & & $\star \star \star$ & \\
\hline & & Bas & ++ & $\star \star \star \star ~$ & +++ & $\star \star \star$ & & & Bas & $* * *$ & & $\star \star \star *$ & \\
\hline \multirow{5}{*}{$\mathrm{V} 2 \mathrm{P} / \mathrm{T} \times \mathrm{V} 3 / 7$} & 1 & Haut & t+t & ++ & ns & $\mathrm{ns}$ & \multirow{5}{*}{$\begin{array}{l}R 2 / T \\
x \\
V 1 / T, V 2 / T \\
\text { et } V 3 / T\end{array}$} & \multirow[t]{3}{*}{1} & Haut & ns & & ns & \\
\hline & & Bas & ++ & ++ & ns & ns & & & Bas & ns & & ns & \\
\hline & & & & & & & & & & & & & \\
\hline & \multirow{2}{*}{2} & Haut & $t+t$ & ++ & +++ & ns & & 2 & Haut & ns & & +++ & \\
\hline & & Bas & +++ & ns & +++ & ns & & & Bas & ns & & ns & \\
\hline \multirow{5}{*}{$\mathrm{V} 1 / \mathrm{T} \times \mathrm{R} 1 / \mathrm{T}$} & \multirow[t]{2}{*}{1} & Haut & $* * *$ & & $* * *$ & & \multirow{5}{*}{$\mathrm{R} 1 / \mathrm{T} \times \mathrm{R} 2 / \mathrm{T}$} & \multirow[t]{2}{*}{1} & Haut & ns & & ns & \\
\hline & & Bas & 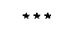 & & ns & & & & Bas & ns & & ns & \\
\hline & & & & & & & & \multirow{3}{*}{2} & & & & & \\
\hline & \multirow[t]{2}{*}{2} & Haut & $\star \star \star *$ & & $\star \star \star \star$ & & & & Haut & * & & $\star \star \star$ & \\
\hline & & Bas & $* \star *$ & & *** & & & & Bas & $*$ & & ns & \\
\hline
\end{tabular}

Pousses $(P): V$, végétatives. Fonctions: $V 1 / T$, leuilles; $V 2 P / T$, masse des axes ; $V 3 / T$, bourgeons ; R1/T, production femelle; R2/T, production mâle. Âge : 1 , arbres de 100 ans de La Dauphine ; 2 , vieux arbres du Plo du Four. + corrélation positive ; * corrélation négative ; +++ ou ${ }^{* *} P<0,001 ;++$ ou ${ }^{* *} 0,001<P<0,01 ;+$ ou * $0,01<P<$ 0,05 ; ns : non significatif.

\section{DISCUSSIONS}

D'une manière générale, cette analyse confirme nos résultats précédents (Thiébaut et Comps, 1991):

- i) les écarts significatifs entre pousses longues et courtes mettent en évidence une certaine autonomie de stratégie de développement dans chacun de ces types. $\dot{A}$ un autre niveau d'organisation, une semiautonomie des branches a déjà été montrée chez certains arbres (Hansen, 1971; Larson, 1980 ; Larson et al, 1980) ;

- ii) dans les pousses hermaphrodites, il y a une synergie souvent significative entre fonctions végétatives, aussi bien au niveau des acquisitions que des allocations alors que dans les pousses végétatives la syner- 
gie demeure pour les acquisitions mais une compétition se dessine pour les allocations ;

- iii) les pousses hermaphrodites présentent des ressources plus importantes que les végétatives, une grande partie de ces ressources étant employée à la reproduction. Une compétition forte se manifeste pour les allocations, le plus souvent entre la fonction femelle et les fonctions végétatives. Ces résultats confirment l'hypothèse d'une compétition directe chez les arbres entre fonctions reproductrices et végétatives pour se partager des ressources limitées (Tuomi et al, 1982 ; Caeser et McDonald, 1983 ; Gartner, 1988).

Cependant, les résultats obtenus ici montrent que les conclusions précédentes doivent être nuancées en fonction de la situation des pousses dans la couronne des arbres et de l'âge de ces derniers. Divers auteurs ont montré qu'au cours de leur développement les arbres passent par différentes phases de croissance. En particulier, lorsque la maturité est atteinte, la croissance en longueur des pousses au sommet du houppier et par conséquent l'incrément en hauteur décroissent après être passés par un maximum (Hallé et al, 1978). Thiébaut et al (1981) puis Thiébaut et Puech (1984) ont décrit plusieurs étapes successives dans le développement d'une branche de hêtre : phase d'exploration dans plusieurs directions (branche $n$-ramifiée à 2-ramifiée), phase d'exploration dans une direction (branche 1-ramifiée), phase de stabilisation (branche non ramifiée) et, enfin, phase d'élagage. Et ces auteurs ont repéré plusieurs zones de bas en haut dans le houppier du hêtre, en relation avec l'âge de l'arbre et selon la phase de développement atteinte par les branches maîtresses, respectivement zone en ramification active, zone en élongation, zone stabilisée puis zone élaguée. Par la suite, Roloff (1989a) a pu établir une relation entre ces modes de développement dans la flèche et le degré d'agression par des agents atmosphériques polluants (Roloff, 1989b) ou des facteurs écologiques défavorables (Dobler et al, 1988). D'autres études ont mis en évidence l'évolution de divers caractères des arbres au cours de leur vie, marquée par un déclin à partir d'un âge qui varie en fonction de l'espèce : évolution du diamètre de la tige chez le noyer (Gannibal et al, 1989) ou de plusieurs caractères en particulier de la masse d'aiguilles produites chez l'épicéa (Mikshis et Ozolinchyus, 1987). Or ces différences fonctionnelles, entre arbres d'âges différents ou entre secteurs d'un même arbre, se traduisent également en termes d'acquisition et d'allocation de matière sèche aux divers types de pousses et aux diverses fonctions végétatives et reproductrices.

Nos résultats révèlent des différences dans l'expression de la vitalité entre les arbres de La Dauphine, plus jeunes, et ceux du Plo du Four, plus âgés. En effet, chez les premiers, le développement végétatif continue à jouer un rôle important : acquisitions aux fonctions végétatives élevées dans les pousses longues, végétatives et florifères; effectif des pousses longues végétatives 3 fois plus fort que chez les vieux arbres; dans les pousses longues, acquisitions aux fonctions végétatives significativement plus grandes dans le haut du houppier à croissance active que dans le bas à croissance ralentie ou stabilisée. Les vieux arbres en revanche sont caractérisés par :

- un faible développement végétatif, plus ou moins stabilisé : pousses longues significativement plus courtes que chez les arbres plus jeunes, aucun écart dans les acquisitions aux fonctions végétatives entre le haut et le bas du houppier ;

- l'envahissement de l'arbre par la floraison: effectif des pousses courtes florifères 2 fois plus élevé qu'à La Dauphine, effort 
prépondérant à la fonction reproductrice femelle.

Ces résultats corroborent un certain nombre d'idées. L'existence d'un rythme endogène de la floraison lié à la maturité des individus, partiellement indépendant des conditions du milieu, a été souvent souligné (Koriba, 1958; Wareing, 1959; Scarrone, 1969 ; Putz, 1979 ; Borchert, 1976 et 1980 ; Puig, 1981 ; Michaloud, 1988). L'aptitude d'une plante à fleurir dépend de l'existence d'une maturation végétative. Chez les espèces polycarpiques, le développement végétatif se poursuit après la première floraison et l'adulte peut fleurir plusieurs fois. Une fois apparue, la floraison tend à occuper une place de plus en plus grande chez l'individu (Hallé et Oldeman, 1970 ; Edelin, 1977 et 1984 ; Barthélémy, 1988), y compris chez le hêtre (Schaffalitzky de Muckadell, 1954 et 1959 ; Thiébaut, 1981; Thiébaut et al, 1981 ; Thiébaut et Puech, 1984). Cet envahissement par la floraison se déroule de façon continue avec l'âge jusqu'à la mort de l'individu. Nos résultats confirment les observations précédentes et apportent 2 précisions supplémentaires :

- données quantitatives entre 2 stades d'adultes à des âges différents ;

- un rapport floraison mâle et femelle se dessine, également sous l'influence de l'âge, chez le hêtre, espèce monoïque aux fleurs unisexuées. La floraison femelle est décalée par rapport à la floraison mâle ; elle arrive plus tard dans la vie de l'arbre et semble devenir envahissante après la floraison mâle.

Enfin ces résultats confirment en termes d'acquisitions chez les arbres adultes en pleine vitalité: le rôle charpentier des pousses longues, en particulier des végétatives, le fonctionnement différentiel en élongation du sommet de la couronne, le rôle non charpentier des pousses courtes alors que les arbres les plus vieux mon- trent bien la tendance à l'homogénéisation du houppier et donc à la stabilisation de l'ensemble de la couronne.

En termes d'allocations, les écarts selon l'âge des arbres dans les pousses florifères sont liés à la distribution respective entre fonctions florifères et végétatives, ce qui confirme les observations similaires en terme de production de Thiébaut et al (1981). Les arbres plus jeunes de La Dauphine, malgré une bonne fructification, privilégient les allocations aux fonctions végétatives alors que les plus vieux allouent très fortement à la reproduction et cela même dans les pousses longues femelles qui ne se comportent plus comme des pousses longues végétatives. Dans les pousses courtes hermaphrodites, la compétition entre reproduction et croissance n'est significative $(P<0,001)$ que chez les arbres âgés. Dans les pousses longues végétatives, l'effort à la croissance est favorisé par rapport à la maintenance chez les arbres de La Dauphine et plus particulièrement au sommet de leur houppier, ce qui confirme les résultats précédents. L'allocation très forte à la maintenance et les corrélations négatives très significatives entre cette fonction et les fonctions de croissance dans les pousses végétatives courtes des 2 groupes d'arbres confirme leur rôle essentiel d'exploitation du milieu.

Le niveau quantitatif d'acquisitions aux différentes fonctions varie souvent d'un arbre à l'autre dans une même classe d'âge ; le même phénomène peut être également observé au niveau des allocations mais à un degré moindre car les écarts inter-groupes sont plus élevés que les écarts intra-groupe. Ces différences mettent en évidence l'existence d'une réponse individuelle, donc à déterminisme en partie d'origine génétique, à des conditions semblables de milieu, comme l'ont déjà souligné Jacquard et Heim (1983) et Jacquard et al (1985) chez Dactylis glomerata. 


\section{REMERCIEMENTS}

L'installation du dispositif expérimental et la récolte des échantillons ont pu être réalisées grâce à la participation de la Direction régionale de Languedoc-Roussillon qui nous a prêté une nacelle hydraulique. Nous avons également bénéficié de laide de $\mathrm{P}$ Baradat pour le traitement des données et de l'aide technique de Mme RM Guilbaud. Nous leur exprimons nos remerciements.

\section{RÉFÉRENCES}

Abrahamson WG, Mc Crea KD (1985) Seasonal nutrients dynamics of Solidago altissima (Compositeae). Bull Torr Bot Club 112, 414420

Agren $J$ (1988) Sexual differences in biomass and nutrient allocations in the dioecious Rubus chamaemorus. Ecology 69, 962-973

Baradat $P$ (1989) Amélioration génétique des arbres forestiers, éléments méthodologiques. Document INRA, Bordeaux

Barthélémy $D$ (1988) Architecture et sexualité chez quelques plantes tropicales : le concept de floraison automatique. Thèse d'Université, Montpellier

Bell KL, Hiatt HD, Niles WE (1979) Seasonal changes in biomass allocation in eight winter annuals of the Mojave Desert. J Ecol 67, $781-787$

Benner BL, Bazzaz FA (1988) Carbon and mineral element accumulation and allocation in two annual plant species in response to timing of nutrient addition. Ecology 76, 19-40

Borchert R (1976) Differences in shoot growth patterns between juvenile and adult trees and their interpretation based on system analysis of trees. Actae Hortic 56, 123-130

Borchert R (1980) Phenology and ecophysiology of tropical trees: Erythrinia poeppigiana OF Cook. Ecology 61, 1065-1074

Brett DW (1963) The inflorescence of Fagus and Castanea, and the evolution of the cupules of the Fagaceae. New Phytol 63, 96-118

Caeser JC, Mc Donald AD (1983) Shoot development in Betula papyrifera. II. Comparison of vegetative and reproductive short shoot growth. Can J Bot 61, 3066-3071
Colombo-Mariani P (1971) Studio fisio-ecologico sul faggio (Fagus sylvatica $\mathrm{L}$ ) dell'altipiano del Cansiglio (Prealpi venete). Arch Bot Biogeogr Ital 47, 1-20

Dina SJ, Klikoff LG (1974) Carbohydrate cycle of Plantago insularis var fastigiata, a winter annual from the Sonoran Desert. Bot Gaz $135,13-18$

Dobler D, Hohloch K, Lisbach B, Saliari M (1988) Trieblangen-Messungen an Buchen. Allgem Forstzschr 29, 811-812

Edelin C (1977) Images de l'architecture des Conifères. Thèse de $3^{e}$ cycle, Montpellier

Edelin C (1984) L'architecture monopodiale: l'exemple de quelques arbres d'Asie tropicale. Thèse de doctorat d'État, Montpellier

Escarré J, Houssard C (1989) Variations de populations de Rumex acetosella $\mathrm{L}$ le long d'une succession secondaire. I. Allocation de biomasse. Acta CEcol 10, 3-19

Gannibal BK, Lovelius NV, Khalimov A (1989) Dynamics of the radial increment of Juglans regia in the western Darvaz (Tajikistan) (en russe). Botanicheskii Zhurnal 74, 227232

Gartner EJ (1988) Operational forest decline symptomatology. In: Scientific basis of forest decline symptomatology. CEE and Institute of Terrestral Ecology (JN Cape, P Mathy, ed) Edinburgh, 295-306

Hallé F, Oldeman RAA (1970) Essai sur l'architecture et la dynamique de croissance des arbres tropicaux. Masson, Paris

Hallé F, Oldeman RAA, Tomlinson PB (1978) Tropical trees and forests. An architectural analysis. Springer Verlag, Berlin

Hansen P (1971) 14C-studies on apple trees. II. The early seasonal growth in leaves, flowers and shoots as dependent upon current photosynthates and existing reserves. Physiol Plant 25, 469-473

Harper JL, Ogden J (1970) The reproductive strateg: of higher plants. I. The concept of strategy with special reference to Senecio vulgaris L. J Ecol 58, 681-698

Hickman JC, Pitelka LF (1975) Dry weight indicate energy allocation in ecological strategy analysis of plants. CEcologia 21, 112-121

Jacquard P, Heim G (1983) Demographic strategies and originating environment in disturbance and ecosystems: components of res- 
ponse (HA Mooney, M Godron, eds). Springer Verlag, Berlin, 226-239

Jacquard P, Ibn Tattou M, Maitre JP, Al Sbei R (1985) Allocation des ressources chez quelques végétaux : facteurs de variation. Bull Ecol 16, 35-46

Koriba K (1958) On the periodicity of tree growth in the tropics, with reference to the mode of branching, the leaf-fall and the formation of the resting bud. Garden's Bull Singapore 17, 11-81

Larson PR (1980) Interrelation between phyllotaxis, leaf development and the primarysecondary vascular transition in Populus deltoïdes. Ann Bot 46, 757-769

Larson PR, Isebrands JC, Dickson RE (1980) Sink to source transition of Populus leaves. Ber Dtsch Bot Ges 93, 79-90

Menges ES (1990) Environmental correlations with male, female and clonal biomass allocation in the forest herb, Laportea canadensis. Am Midl Nat 124, 171-180

Michaloud $G$ (1988) Aspects de la reproduction des figuiers monoïques en forêt equatoriale africaine. Thèse d'Université, Montpellier

Mikshis VI, Ozolinchyus RV (1987) The growth and structure of the crowns of open-grown Norway spruce trees (en Russe). Lesovedenie 6, 54-61

Munson AD, Timmer VR (1990) Site specific growth and nutrition of planted Picea mariana in the Ontario clay Belt. III. Blomass and nutrient allocation. Can $J$ For Res 20, 11651171

Nielsen O (1977) Seasonal and annual variation in litter fall in a beech stand 1967-1975. Forstl Forsoegsvaes Dan 35, 16-38

Oswald $H$ (1981) Régénération naturelle, importance et périodicité des fainées. Influence des facteurs climatiques et sylvicoles. In: Le Hêtre ( $E$ Teissier du Cros, $F$ Le Tacon, $G$ Nepveu, J Parde, R Perrin, J Timbal, eds). INRA, départ rech for, Paris, pp 207-216

Pitelka LF (1977) Energy allocation in annual and perennial lupines (Lupinus: Leguminosae). Ecology 58, 1055-1065

Puig $H$ (1981) Rythmes de floraison, de fructification et de défoliation d'Eperua falcata Aubl en forêt guyanaise. In: Actes du 106 Congrès Nat Sociétés savantes, Perpignan, p 139-148
Putz FE (1979) A seasonality in Malaysian tree phenology. Malays For 42, 1-23

Rameau C (1986) Allocations des ressources à la croissance, la reproduction et la survie chez le glaïeul. Thèse d'Université, Montpellier

Roloff A (1989a) Entwicklung und Flexibilität der Baumkrone und ihre Bedeutung als VItalitätsweisen. Schweiz Zschr Forstwesen 140, 775789, 943-963

Roloff A (1989b) Kronenarchitektur als Zeichen der Baumvitalität bei Laubbäumen. Gartenamt 38, 490-496

Scarrone F (1969) Recherches sur les rythmes de croissance du manguier et de quelques végétaux ligneux malagasy. Thèse de doctorat d'Etat, Clermont-Ferrand

Schaffalitzky de Muckadell M (1954) Juvenile stages in woody plants. Physiol Plant 7, 782796

Schaffalitzky de Muckadell M (1959) Investigations on ageing of apical meristems in woody plants and its importance in sylviculture. Forstl Forsogsvags Dan 25, 310-455

Staaf $H$, Jernquist I (1986) Seasonal dynamics especially autumnal retranslocation of nitrogen and phosphorus in foliage of dominant and suppressed trees of beech, Fagus sylvatica L. Scan J For Res 1, 333-342

Thiébaut $B$ (1981) Formation des rameaux chez le hêtre. In: Le Hêtre (E Teissier du Cros, $F$ Le Tacon, G Nepveu, J Pardé, R Perrin, J Timbal, eds), INRA, départ rech forest, Paris, 169-174

Thiébaut B (1982) Observations sur le développement de plantules de hêtre (Fagus sylvati$c a$ L) cultivées en pépinière, orthotropie et plagiotropie. Can J Bot 60, 1292-1310

Thiébaut B, Payri C, Vigneron P, Puech S (1981) Observations sur la croissance et la floraison du hêtre. Naturalia Monspeliensia 48, 1-25

Thiébaut B, Puech S (1983) Développement du hêtre commun (Fagus sy/vatica L), morphologie et architecture de l'arbre. I. Le développement des plants. Rev For Fr 35, 443-451

Thiébaut B, Puech S (1984) Développement du hêtre commun (Fagus sylvatica L), morphologie et architecture de l'arbre. It. Le développement des arbres. Rev For Fr 36, 45-58

Thiébaut B, Comps B (1991) Répartition de la matière sèche entre les organes végétatifs et reproducteurs dans les pousses annuelles du 
hêtre (Fagus sylvatica L). Can J Bot 69, 2225-2231

Tuomi J, Niemela P, Mannila R (1982) Resource allocation on dwarf shoots of birch (Betula pendula): reproduction and leaf growth. New Phytol 91, 483-487

Van Baalen J, Ernst WHO, Van Andel J, Janssen DW, Nelissen HJM (1990) Reproduction allocation in plants of Scrophularia nodosa grown at various levels of irradiance and fertility of soil. Acta Bot Neerl 39, 183-196

Wall DA, Morrison IN (1990) Phenological development and biomass allocation in Silene vul- garis (Moench) Garcke. Weed Res 30, 279288

Wankhar B, Tripathi RS (1990) Growth and reproductive allocation pattern of Centella asiatica raised from stem cuttings of different sizes in relation to light regimes, soil texture and soil moisture. Acta CEcol 11, 683-692

Wareing PF (1959) Problems of juvenility and flowering in trees. J Linn Soc London Bot 56 , 282-289

Williams RB, Bell KL (1981) Nitrogen allocation in Mojave Desert winter annuals. CEcologia $48,145-150$ 